\title{
Brain Connectivity in Disorders of Consciousness
}

\author{
Mélanie Boly, ${ }^{1}$ Marcello Massimini, ${ }^{2}$ Marta Isabel Garrido, ${ }^{3}$ Olivia Gosseries, ${ }^{1}$ \\ Quentin Noirhomme, ${ }^{1}$ Steven Laureys, ${ }^{1}$ and Andrea Soddu ${ }^{1}$
}

\begin{abstract}
The last 10 years witnessed a considerable increase in our knowledge of brain function in survivors to severe brain injuries with disorders of consciousness (DOC). At the same time, a growing interest developed for the use of functional neuroimaging as a new diagnostic tool in these patients. In this context, particular attention has been devoted to connectivity studies - as these, more than measures of brain metabolism, may be more appropriate to capture the dynamics of large populations of neurons. Here, we will review the pros and cons of various connectivity methods as potential diagnostic tools in brain-damaged patients with DOC. We will also discuss the relevance of the study of the level versus the contents of consciousness in this context.
\end{abstract}

Key words: connectivity; consciousness; diagnosis; minimally conscious state; neuroimaging; vegetative state

\section{Introduction}

$\mathbf{T}$ HIS REVIEW ATTEMPTS to discuss the different approaches that may be used to investigate connectivity in patients with disorders of consciousness (DOC). We will first define the different common clinical entities considered as pathological DOC. We will then discuss the possible approaches that can be used to study brain connectivity in these severely brain-damaged patients. We will then consider conceptual differences between the study of neural correlates of consciousness (NCC) in DOC and in healthy awake volunteers (HV)-referring to the study of the level versus the contents of human consciousness. Finally, we will briefly discuss the need for the validation of different diagnostic techniques, before their application at the patients' bedside, and review potential steps toward achieving this goal.

DOC remain among the most poorly understood and less treatable conditions of modern neurology. They are commonly divided into three main clinical entities. Coma is defined by a lack of both arousal and awareness. Vegetative state (VS) is defined by a lack of behavioral signs of consciousness, while arousal (defined as an alternation of eye opening and closing) is preserved (Laureys et al., 2004). In contrast, a minimally conscious state (MCS) is defined by the presence of nonreflexive behaviors, although patients remain unable to communicate (Giacino et al., 2002). Recent studies showed that in the absence of use of an appropriate clinical scale such as the Coma Recovery Scale-Revised
(CRS-R), the risk of underestimating the level of consciousness in DOC patients is about 40\% (Schnakers et al., 2009). This fact illustrates the difficulties inherent to the behavioral assessment in these patients. Many patients' behaviors, such as grimacing or localization of sounds, for example, remain equivocal and difficult to interpret as evidence for the presence or absence of consciousness. Research, therefore, aims at finding reliable brain activity markers of consciousness that are applicable to these patients. Finding such markers would improve both diagnosis and disease understanding, bringing possibilities for the setup of new treatments (Boly, 2011). Functional neuroimaging studies performed in unresponsive brain-damaged patients can be categorized into two main classes: active and passive paradigms (Boly and Seth, 2012). Active paradigms aim at obtaining a response to command in patients, while bypassing the motor response itself (Boly et al., 2007; Owen et al., 2006). Passive paradigms gather information on the patients' brain function, identifying neural activation reflecting "automatic" processing, that is, occurring without the patients' willful intervention (Boly et al., 2009a). [18F]-fluorodeoxyglucose positron emission tomography (PET) studies showed a widespread impairment of fronto-parietal cortices in VS patients as compared with controls (Laureys, 2005; Laureys et al., 1999). Although metabolic studies certainly provide valuable information, connectivity may, however, better grasp the neurons' collective behavior. Brain connectivity can be studied at both the structural (Fernandez-Espejo et al., 2011) and functional (Noirhomme

\footnotetext{
${ }^{1}$ Coma Science Group, Cyclotron Research Centre and Neurology Department, University of Liège and CHU Sart Tilman Hospital, Liège, Belgium.

${ }^{2}$ Department of Clinical Sciences, "Luigi Sacco" University of Milan, Milan, Italy.

${ }^{3}$ Wellcome Trust Centre for Neuroimaging, Institute of Neurology, University College London, London, United Kingdom.
} 
et al., 2010; Soddu et al., 2009, 2011) levels. Functional studies are not necessarily trying to univocally reflect structural connectivity. Although recent attempts at predicting functional from structural connectivity are very promising (Honey et al., 2007), some discrepancies may exist when comparing the predicted functional connectivity with the empirical results obtained by measuring the resting-state blood-oxygenlevel-dependent (BOLD) signal through functional magnetic resonance imaging (fMRI). The present review will focus on the functional aspects.

On the functional side, it may be useful to introduce here some relevant concepts that will be further discussed. Functional connectivity measures the deviations from statistical independence among distant neural populations. Statistical dependence is estimated based on temporal relationships, by applying nondirected (for example, linear correlation and coherence-based analyses) or directed (Granger causality and transfer entropy) measures to time series of neuronal activity (Bressler and Seth, 2011). Measures of functional connectivity are largely model free but do not make any explicit reference to the underlying structural model. Hence, for instance, functional connectivity among distant elements may be high either because these elements are actually connected by direct structural links or just because they share a common input. On the other hand, effective connectivity attempts to extract networks of causal effects among neural elements. Causal effects can be inferred directly by detecting the large-scale effects produced by a controlled (peripheral, cortical-electric, and corticalmagnetic) perturbation of the system. Alternatively, patterns of effective connectivity can be extracted from spontaneous or evoked activity by means of dynamic causal modeling (DCM). This approach requires specifying a model, including structural parameters, that informs on the original neuronal mechanism at the basis of the observed signals. The next section will review how these different approaches to the study of connectivity may be employed in DOC patients.

\section{Studying Brain Connectivity in DOC-the Different Approaches}

To date, three different connectivity approaches have been used in patients with DOC: (1) functional connectivity or "resting state" studies using, for example, fMRI; (2) a perturbational approach using transcranial magnetic stimulation (TMS) combined with electroencephalography (EEG); and (3) other perturbational approaches investigating brain responses to external stimuli, including sensory evoked potentials (ERP). After summarizing the findings obtained in DOC with each of these techniques, we will review their respective practical strengths and weaknesses. Figure 1 condenses the main concepts of the present section.

\section{Functional connectivity studies}

Functional connectivity investigates linear relationships among the measured activities in the different regions of interest of the brain. Both univariate, comparing each time pairs of regions, and multivariate, assessing all the regions at the same time, approaches are nowadays available, each

Resting state fMRI
- Whole brain coverage
- High spatial resolution
-Efficient study of cortical
structures
-Easy to acquire in clinical
settings
- Low temporal resolution
- Prone to noise
contamination
-Significance unknown
- Functional connectivity is
prone to third driver effects

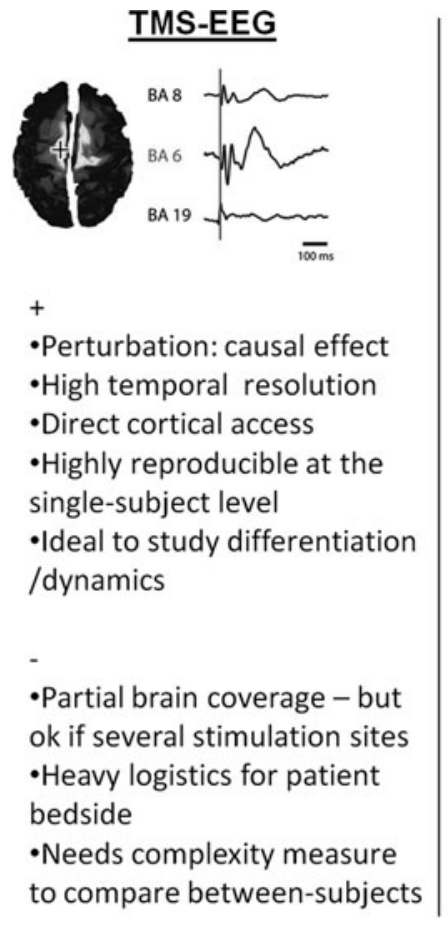

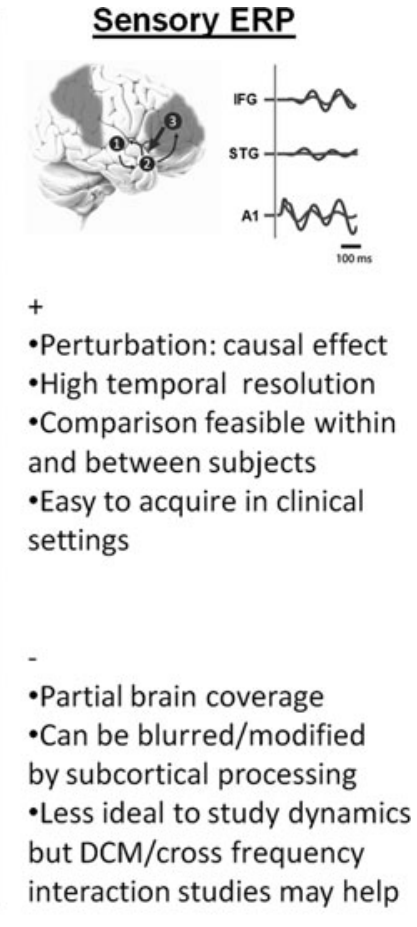

FIG. 1. Pro (+) and cons (-) of different approaches applicable to the study of brain connectivity in patients with disorders of consciousness. Detailed description can be found in the main text. Upper panels are reproduced with permission from (Boly et al., 2011; Massimini et al., 2009; Vanhaudenhuyse et al., 2010). fMRI, functional magnetic resonance imaging; TMS, transcranial magnetic stimulation; EEG, electroencephalography; ERP, evoked potentials; DCM, dynamic causal modeling; BA, Brodmann area; A1, primary auditory cortex; STG, superior temporal gyrus; IFG, inferior frontal gyrus. Right panel: brain area $1=\mathrm{A} 1$, area $2=$ STG , area $3=$ IFG. Arrows represents connection between the different network nodes as for example studied by DCM. 
with its own advantages and limitations. Functional connectivity can, in theory, be applied to a variety of brain imaging techniques. Resting-state fMRI functional connectivity has proved useful in the identification of a correlation between the fronto-parietal networks connectivity and the level of consciousness in DOC patients (Boly et al., 2009b; FernandezEspejo et al., 2010; Vanhaudenhuyse et al., 2010) or anesthesia (Boveroux et al., 2010; Martuzzi et al., 2010; Schrouff et al., 2011). Some decreases in fronto-parietal connectivity have been described in deep nonrapid eye movement (non-REM) sleep (Horovitz et al., 2009; Samann et al., 2011). Other studies, however, show globally preserved connectivity across sleep stages (Koike et al., 2011). Anticorrelations between a default network and lateral fronto-parietal cortices have also been shown to decrease in VS (Boly et al., 2009b), anesthesia (Boveroux et al., 2010), and non-REM sleep (Samann et al., 2011) as compared with normal wakefulness.

Although this technique is very useful in collecting information about whole-brain function in one single data set, the functional significance of resting-state fMRI signals has been debated (Birn et al., 2008). It is now commonly accepted that the BOLD signal tightly correlates with population-level neuronal activity, especially with regard to local field potentials (Nir et al., 2007, 2008). The relationship between the BOLD signal and regional electrophysiological activity is likely to be similar for task-related fMRI or the resting state. Consequently, the functional significance of spontaneous or task-evoked BOLD fluctuations is also likely to be similar. Resting-state and task-related fMRI are prone to noise contamination, such as by movement and physiological artifacts, which are difficult to avoid in HV as in DOC patients (Soddu et al., 2011, 2012). Indeed, DOC patients are noncollaborative and may present uncontrollable reflexive movements. Even if different methods exist for dealing with them (Fox et al., 2005; Jones et al., 2008; Perlbarg et al., 2007; Soddu et al., 2012), none of them are completely satisfactory. fMRI temporal resolution is also slower than the temporal scale where conscious scenes are likely to arise. This temporal scale is indeed thought to be of the order of hundreds of milliseconds, rather than seconds. fMRI studies could, thus, be usefully complemented by EEG.

Resting-state spontaneous brain activity analyses can also be performed using EEG. The loss of consciousness during non-REM sleep or anesthesia is usually associated with a raise of delta power and the occurrence of slow waves in the EEG (Murphy et al., 2011). These slow waves travel across a wide range of brain areas, especially in the default network (Murphy et al., 2009, 2011). The bispectral index is a commercial EEG-based measure that is mainly based on power spectral changes in the EEG between different states, and for small value ranges is largely based on changes in power in the delta band. The fact that the bispectral index can differentiate between MCS and VS patients (Schnakers et al., 2008) also suggests a negative correlation between the EEG delta power and the level of consciousness in DOC patients. A small number of studies have described decreased scalp EEG coherence in coma (Kane et al., 1998), and in one VS (Davey et al., 2000) and one MCS (Kobylarz and Schiff, 2005) patient. Recent reports have, however, described increased EEG coherence during propofol-induced loss of consciousness (Murphy et al., 2011; Supp et al., 2011). Granger causality estimates causal statistical influences between simultaneously recorded
EEG time series data, either in the absence of identifiable behavioral events or in the context of task performance. Granger causality measures between default network areas and signals reconstructed from spontaneous high-density EEG recordings have been reported to be increased during propofol-induced loss of consciousness (Barrett et al., 2012). On the other hand, connectivity assessment using Transfer Entropy, a method used to measure directional information transfer between the EEG signals recorded from different scalp electrodes (Schreiber, 2000), suggests a decrease of front-to-back connectivity in this state (Ku et al., 2011; Lee et al., 2009). This result suggests a possible impairment of top-down cerebral processes during propofol sedation. EEG coherence and Granger Causality connectivity also seem decreased in MCS patients as compared with severely disabled patients who emerged from DOC (Pollonini et al., 2010). Further studies using, for example, intracranial data are warranted to complement these findings.

The limitation of spontaneous brain activity connectivity measures (and related graphical approaches) of not being able to infer the underlying neuronal mechanism can be partially resolved by the use of mathematical models such as DCM (Friston et al., 2003). Correlation-based measures and their variants are also prone to third driver effects, that is, they cannot differentiate between direct causal interactions in brain areas and the influence of a third common generator driving the observed correlation, which would be located somewhere else in the brain. Perturbational approaches may help in reducing this bias by recording brain activity changes directly due to experimentally induced causal neuronal interactions.

\section{Perturbational approach I: TMS-EEG studies}

Consciousness is considered as emerging from fast, effective interactions among distributed areas, especially within a cortico-thalamic neuronal complex (Tononi, 2008). One way of effectively gauging connectivity within human thalamo-cortical networks involves directly perturbing a subset of cortical neurons with TMS and performing the source modeling of high-density EEG data to detect, on a millisecond time scale, the chain of effects triggered in the rest of the brain by this initial perturbation (Casali et al., 2010; Ilmoniemi et al., 1997; Litvak et al., 2007; Morishima et al., 2009; Stamm et al., 2011). Since TMS tends to activate a large set of cortical axons in a way that is difficult to fully control (Wagner et al., 2007), TMS-EEG is not ideally suited for capturing causal interactions within specific cortico-cortical circuits. On the other hand, TMS-evoked activations provide an overall, broad estimation of the effective connectivity that may constitute an advantage when studying DOC; indeed, theoretical works (Tononi, 2004; Tononi and Koch, 2008), experimental data (Alkire et al., 2008), and clinical evidence (Markowitsch and Kessler, 2000; Mataro et al., 2001; Schiff, 2010) suggest that consciousness does not depend so much on some specific circuits, but rather on the capacity of distributed regions of the brain to interact through divergent cortico-cortical and cortico-thalamo-cortical connections. Thus, the combination of TMS and EEG was tested in different conditions in which consciousness was physiologically or pharmacologically altered. First, TMS-evoked cortical activations were recorded during the transition from wakefulness to deep non-REM 
sleep early in the night, when consciousness fades. These measurements showed that, while during wakefulness the brain is able to sustain long-range, complex patterns of activation, during non-REM sleep, this ability is lost: The thalamocortical system, despite being active and reactive, either breaks down into causally independent modules (producing a local response) (Massimini et al., 2005), or bursts into an explosive and nonspecific response (producing a simple, global slow wave that invades most of the cortex) (Bergmann et al., 2012; Massimini et al., 2005, 2007). In a subsequent study, TMS-evoked activations were recorded during REM sleep, when the brain is still disconnected from the external world but consciousness returns in the form of a dream (Massimini et al., 2010). In this condition, TMS-EEG responses were similar to the ones observed during wakefulness. Finally, the spread of TMS-evoked cortical activations was studied during LOC induced by a pharmacological agent, midazolam, at anesthetic concentrations (Ferrarelli et al., 2010). In this case, unconsciousness was associated with a breakdown of the long-range pattern of cortical effective connectivity. Based on these findings TMS-EEG has been proposed as a tool to investigate effective connectivity at the bedside of DOC patients (Massimini et al., 2009). In this category of patients, TMS-EEG may be especially useful, because it does not rely on a subject's ability to process sensory stimuli, to understand and follow instructions, or to communicate. Notably, a recent TMS-EEG study demonstrated a clear-cut recovery of effective connectivity in the brain of noncommunicating patients that paralleled the recovery of consciousness from the VS (Rosanova et al., 2012). Practically, TMS-EEG is still a rather challenging technique that requires the implementation of a complex set-up in the intensive care environment. Hence, simpler ways of estimating effective connectivity in DOC patients should be developed in parallel; this could be achieved by using standard sensory stimulation.

\section{Perturbational approach II: response to external stimuli}

Responses to external stimuli have been studied for quite a while in patients with DOC. The first $\mathrm{H}_{2}{ }^{15} \mathrm{O}$-PET studies performed in DOC patients, measuring changes in regional cerebral blood flow, showed that at the group level, VS activation in response to simple auditory and somatosensory stimulation was restricted to primary sensory cortices (Laureys et al., 2000, 2002). Patients in MCS showed a more widespread activation, including fronto-parietal cortices, as well as default network deactivation, in response to external stimuli (Boly et al., 2004, 2008a). fMRI studies showed the presence of the activation of higher-order cortices in some VS patients, which is often correlated with a good prognosis (Coleman et al., 2009; Di et al., 2007).

Recent EEG studies suggested the importance of longlatency components (of more than 100-200 msec delay after the onset of stimuli) in normal conscious perception (Del Cul et al., 2007; Fitzgerald et al., 2001). The amplitude of these long-latency components indeed selectively increases with the probability of perception of a given stimulus. This amplitude also correlates with the level of consciousness in VS and MCS patients (Boly et al., 2011), during propofol anesthesia (Heinke et al., 2004) or during non-REM sleep (Ruby et al., 2008). Previous studies using DCM in HV suggested a privileged role of backward connectivity in the generation of long-latency ERP components (Garrido et al., 2007). Indeed, this work suggests that while the modulation of forward connections is sufficient in a cortical model to explain the generation of the early components of ERP responses, the activity of backward connections is necessary to generate longerlatency responses. Since long-latency components have also been found to correlate with conscious perception, this result suggests that the activity of backward connections could also be important for consciousness. Correspondingly, a recent DCM analysis suggested that backward connectivity from frontal to temporal cortices could be impaired in VS as compared with MCS and controls (Boly et al., 2011). Altogether, these results stress the likely importance of backward connectivity in higher-order cortices for conscious processing (Crick and Koch, 1995; Lamme, 2006). Further studies conducted on sleep and anesthesia, as well as the use of other ERP paradigms (King et al., 2011), will attempt at evaluating the generalizability and diagnostic value of these findings. In addition, other connectivity assessment methods-such as correlation, coherence, Granger Causality, partial directed coherence, or transfer entropy-should also be used to identify dynamical connectivity changes during external stimulation (Bressler and Seth, 2011) in MCS and VS.

The advantages of external stimuli, thus, rely on providing a perturbational approach that investigates cortical connectivity, without the technical burden of TMS-EEG stimulation. ERP amplitudes are more comparable from one subject to another in a between-subject setting, allowing straightforward statistics at the group level (Boly et al., 2011). However, responses to external stimuli may be filtered through afferent pathways and subcortical gates before reaching the thalamo-cortical system. This means, for instance, that brainstem lesions may obliterate response to external stimuli even though thalamo-cortical interactions are not impaired (Massimini et al., 2009). The brainstem function is, however, thought to be essentially preserved in VS and MCS (Laureys et al., 2004).

The temporal blurring of ERP responses to external stimuli-that is, the observed ERP impulse-response duration for a given single input-is much bigger than that of TMSEEG stimuli. This blurring increases the overlap between the responses in the different brain areas. This potentially decreases the utility of auditory ERP that investigates cortical response differentiation, based on a time series examination of brain activity. An alternative method of studying the complexity of brain responses to external stimuli could be, for example, to study the nonlinearity of the brain responses during responses to stimuli. For example, in the presence or loss of cortical differentiation, the activity in one brain area would be expected to elicit a similar brain activity pattern, with a similar frequency content, in surrounding regions (Boly et al., 2009a). In contrast, the observed brain activity pattern would be very different in the presence of differentiated and integrated cerebral activity (Alkire et al., 2008). Investigating cross-frequency interactions between brain regions could, therefore, be another way of investigating the complexity of cerebral activity in response to stimulation (Ahrens et al., 2008). Following this logic, cross-frequency interactions could be expected to decrease in anesthesia, sleep, and DOC. Previous studies suggested the preponderance of nonlinearity in backward connections, as compared with forward 
connections (Chen et al., 2009). The integrity of cerebral backward connectivity could, thus, be hypothesized as being correlated with preserved brain activity complexity in patients with DOC. Further studies should, however, investigate this issue in greater detail.

\section{Diagnosing Consciousness: Level Versus Contents}

Figure 2 summarizes the present section-which will aim at reviewing the conceptual and practical differences between the study of the level versus the contents of human consciousness, and at emphasizing their relevance for the study of brain function in DOC patients.

As previously discussed by several authors in the literature (Baars, 1988; Frith, 2011; Zeman, 2001), consciousness continues to be an ill-defined concept. When scientifically approaching NCC, there is an important distinction to be made between the level and the contents of consciousness (Dehaene and Changeux, 2005; Frith, 2011; Overgaard and Overgaard, 2010). In the present context, consciousness is defined as what disappears during dreamless sleep (Tononi, 2008): the ability to perceive anything. The contents of consciousness refer to any of the particular perceptions that can be experienced during a normal wakefulness state. These contents may be internally driven or generated through an interaction with the environment (Vanhaudenhuyse et al., 2011). The level of consciousness refers to a dimension that varies from coma at one extreme, through sleep, and to alert wakefulness at the other extreme (Frith, 2011). The level of consciousness can in this view be compared with a potential to experience a lot of different contents, though each cognitive or sensory function by itself would not be necessary for the existence of consciousness (Boly and Seth, 2012). By definition, this potential would disappear in unconscious states. Understanding the levels of consciousness is of particular relevance to the studies of DOC patients, as well as to brain function in different stages of anesthesia and sleep. Ethically, detecting a potential ability to perceive - anything - is a very important question in noncommunicative severely braindamaged patients.

Alert wakefulness is characterized by a consciousness of an ever-changing stream of specific mental states-comparable to a wandering exploration of the potential repertoire of states available to our consciousness at each single moment (Sadaghiani et al., 2010). These transient states that we are aware of at each instant determine the contents of our consciousness. In studies of the NCC in HV, there is great interest in contrasting the neural activity associated with stimuli that influence the contents of consciousness with the neural activity associated with the same stimuli when they affect behavior in the absence of any change in the content of consciousness (Frith et al., 1999). The key difference between the study of NCC in HV and DOC patients is, however, that in the first case, subjects are basically conscious all the time-what changes is the content of their experiences at particular experimentally controlled moments (Boly and Seth, 2012). As noted by (Frith, 2011), though a certain minimal level of consciousness is necessary for there to be any contents of consciousness, the level of consciousness does not determine what the contents of consciousness will be. A certain level of consciousness is prerequisite to any perception, but a series of other factors-such as stimulus intensity, duration or emotional valence, underlying brain activity shaped by previous learning, and ongoing spontaneous brain activity fluctuationscan also influence the conscious access of some given stimuli (Boly et al., 2008b; Dehaene and Changeux, 2005). As stated in (Dehaene and Changeux, 2011), theoretical approaches to NCC in HV-that is, NCC of conscious access, or consciousness contents - and in DOC - that is, NCC of the conscious level-are from two different scopes. In principle, theoretical predictions in terms of NCC for these two aspects could, thus, be different.

\section{NCC in altered consciousness states: \# LEVELS (ANY CONTENT)}

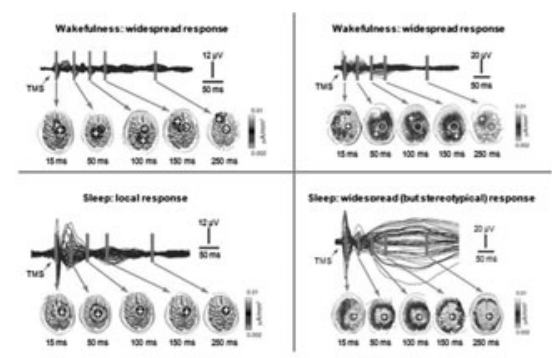

Question: is the subject conscious = able to perceive ANYTHING?

Levels of consciousness are a graded continuum

Study can be dissociated from content: Levels of Consciousness can be efficiently differentiated by subliminal stimuli (TMS-EEG for example)

Correlates described:

- strength of connectivity in the thalamocortical system

- AND complexity/differentiation of the responses

NCC in healthy awake volunteers: LEVEL $=, \neq$ CONTENTS

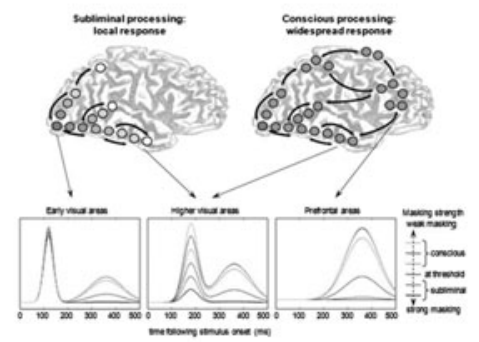

Question: what does the subject perceive = subjects are conscious throughout - but what is the content of their consciousness?

Switching between $\neq$ contents of consciousness can be all or none-concept of a threshold

Study of a particular stimulus response in $\mathrm{HV}$, when the stimulus is perceived consciously as compared to control baseline

Correlates described:

- mainly strength of connectivity ('ignition') in the thalamocortical system
FIG. 2. Conceptual and practical differences in the study of NCC in states such as coma, anesthesia, and sleep, or in healthy awake volunteers. Detailed description can be found in the main text. Upper panels are reproduced with permission from (Alkire et al., 2008; Del Cul et al., 2007).

NCC, neural correlates of consciousness; HV, healthy awake volunteers. 
In practice, there exists some possible dissociation between the experimental study of consciousness level and that of consciousness contents. One can, for example, differentiate various levels of consciousness, without asking any questions about conscious contents. In principle, this question can be addressed via the study of spontaneous brain activity, in an experimental setup lacking control on the subjects' conscious contents. This approach is commonly used in resting-state fMRI or spontaneous EEG acquisition studies. In the context of perturbational approaches, some subliminal stimulations have also been shown to efficiently differentiate the levels of consciousness across different conditions. The TMS-EEG stimulation is typically not perceived by $\mathrm{HV}$ and, in common experimental settings, does not overtly affect the subjects' conscious contents. Despite its subliminal characteristic, TMS responses to stimulation have been shown as efficiently discriminating the levels of consciousness in states such as anesthesia (Ferrarelli et al., 2010), severely brain-damaged patients (Rosanova et al., 2012), or sleep (Massimini et al., 2005). Similarly, simple auditory stimulations such as clicks (Boly et al., 2004) or beeps used in a mismatch negativity paradigm (Boly et al., 2011; Heinke et al., 2004; Ruby et al., 2008) - in which processing is commonly considered to be pre-attentive-could act by probing some basic characteristics of the system that are necessary for some level of consciousness to emerge (Massimini et al., 2009), without necessarily significantly influencing the subjects' conscious contents. Another example can be found in the results of some experiments performed during REM sleep. During this sleep stage, external sounds are usually hardly perceived, but ERP still look very similar to those observed in wakefulness. The observed brain response to auditory stimulation is, thus, likely uncovering a relatively preserved level of consciousness, without being a signature of a modification of its contents (Nir and Tononi, 2010). These different examples show that, in practice, the basic NCC of the level of consciousness can be studied independently of consciousness contents.

As shown in Figure 2 (left panel), the neural correlates of a reduced level of consciousness consist either in a local response to the stimulation (Massimini et al., 2005) or in a global, nonspecific response (Alkire et al., 2008; Kroeger and Amzica, 2007; Massimini et al., 2007). This phenomenon is generally thought to be due to the presence of bistable dynamics in the system (Massimini et al., 2012), which would prevent the emergence of complex spatiotemporal patterns of effective connectivity that are widespread and differentiated at the same time. Similarly, patterns of activity that are widespread, but stereotypical, should be expected during the loss of consciousness in epileptic patients. From this perspective, the level of consciousness seems to correlate better with the complexity of brain activation than with their spatial extent. Thus, quantifying the complexity of brain responses may be a necessary step if one wants to reliably assess the level of consciousness in noncommunicative brain-damaged patients (Boly, 2011; Boly et al., 2009a; Massimini et al., 2009). On the other hand (Fig. 2, right panel), the neural correlates of conscious perception of a specific stimulus in $\mathrm{HV}$ invariably consist of a stronger, and more diffuse, activation for perceived stimuli as compared with subliminal stimuli. This "ignition" process is characterized by the presence of stronger long-range coherence and fast frequency responses involving fronto-parietal cortices for stimuli that reach the threshold for consciousness (Dehaene et al., 2006). In this case, observing a widespread response may immediately signal that a subject consciously perceives the particular stimulus she/he is presented with. In unconscious subjects, it will certainly be important to better understand, and to better conceptualize, the possible dissociations between the neural correlates of the level of consciousness and the neural correlates of a specific aspect of consciousness. If used in severely brain-damaged patients, a single criteria of big response/activation for deciding the presence of consciousness presents, however, a risk of false positive, due to the occurrence of high amplitude responses commonly present in DOC - similar to the K-complex responses observed during non-REM sleep (Boly et al., 2009a). An additional check for the complexity of brain response and the presence of brain activity differentiation would, thus, be a safer bet in order to reliably detect the consciousness in DOC patients (Boly, 2011).

\section{Toward the Use of Neuroimaging as a Diagnostic Test?}

To date, the gold standard for diagnosis of the level of consciousness is behavioral. Clinical assessment is based on criteria described by (Giacino et al., 2002) to distinguish MCS from VS. However, this clinical diagnosis itself has its ownlimitations. If not using an appropriate behavioral scale, clinical misdiagnosis reaches up to $41 \%$ (Schnakers et al., 2009). Clinical scales themselves are quite variable in their reliability. A recent meta-analysis revealed that the CRS-R was the best attempt that explicitly incorporates the diagnostic criteria for MCS in clinical diagnosis (Seel et al., 2010). However, the reliability of the CRS-R itself depends on examiner experience (Lovstad et al., 2010), and this scale is quite long and heavy to administer in a routine basis in acute patients. Even if this gold-standard behavioral assessment is repetitively and carefully performed in DOC patients, it can also sometimes lead to some underestimation of the patients' level of consciousness. It has indeed been shown that a minority of patients who are behaviorally totally unresponsive instead become conscious when assessed with active paradigms using fMRI (Bardin et al., 2011; Monti et al., 2010; Owen et al., 2006) or EEG (Cruse et al., 2011). On the other hand, the active neuroimaging paradigms used to detect these patients are also prone to false negatives (Bardin et al., 2011). In fact, in principle, paradigms that are based on response to command, being clinical or neuroimaging based, are not sufficient to systematically detect consciousness in each individual case (Boly, 2011). Indeed, volition does not equate consciousness: The absence of a response from the patient does not necessarily mean that the patient is unconscious (Boly et al., 2007) and even in HV, volitional activity and consciousness can, in many cases, be dissociated (Boly and Seth, 2012; Soon et al., 2008). Recent results obtained using the isolated forearm technique during anesthesia (Russell and Wang, 1996) raise even more debate on this topic, by showing frequent responses to command in the absence of a subsequent report. To date, there is also a need to fill the gap between clinical behavioral studies and neuroimaging studies by comparing the results obtained using these two approaches on a single-subject basis and identifying typical VS and MCS patterns, versus outlier patterns. Different neuroimaging 
techniques could, thus be compared in terms of their performance-false-positive and false-negative rates, sensitivity, specificity, and so on-if used in sufficiently large populations. Inter-assessor and inter-site reliabilities should also be assessed in order to evaluate the reproducibility of different techniques results on a larger scale. Finally, the use of automated procedures (Soddu et al., 2012), as well as the use of classifiers (Phillips et al., 2011), and of a combined study of different imaging techniques (Bruno et al., 2011) could also reduce observer-dependent bias in neuroimaging-based diagnosis.

Ultimately, a most accurate diagnosis of consciousness would require first knowing the mechanisms bridging conscious perception to the brain and then using neuroimaging techniques as a way of objectifying the presence of this mechanism. To achieve this aim, theoretical approaches attempting at describing the general mechanism underlying conscious experiences (Dehaene and Changeux, 2011; Tononi, 2008), as well as the potential use of theoretically grounded indexes for the neuroimaging-based diagnosis of consciousness (Seth et al., 2008, 2011), are of great interest. However, a lot more work is needed in this field, and we are still a long way from bringing these theories to truly testable predictions (Boly and Seth, 2012). Meanwhile, an NCC-based assessment approach, optimally using the vast information already present in the literature concerning brain function in different DOC states, may provide valuable information about the potential ability of a noncommunicative patient's brain to allow consciousness-information that could usefully complement the clinical diagnosis.

\section{Acknowledgments}

This work was supported by the Belgian Fonds National de la Recherche Scientifique(FNRS), European Commission, Mind Science Foundation, McDonnell Foundation, French Speaking Community Concerted Research Action (ARC 06/ 11-340), and Fondation Léon Frédéricq. O.G. is Research Fellow, M.B. and Q.N. are Postdoctoral Fellows, and S.L. is Senior Research Associate at the FNRS. M.G. is supported by the Wellcome Trust.

\section{Author Disclosure Statement}

The authors declare no conflict of interest.

\section{References}

Ahrens MB, Linden JF, Sahani M. 2008. Nonlinearities and contextual influences in auditory cortical responses modeled with multilinear spectrotemporal methods. J Neurosci 28:1929-1942.

Alkire MT, Hudetz AG, Tononi G. 2008. Consciousness and anesthesia. Science 322:876-880.

Baars BJ. 1988. A Cognitive Theory of Consciousness. Cambridge: Cambridge University Press.

Bardin JC, Fins JJ, Katz DI, Hersh J, Heier LA, Tabelow K, et al. 2011. Dissociations between behavioural and functional magnetic resonance imaging-based evaluations of cognitive function after brain injury. Brain 134:769-782.

Barrett AB, Murphy M, Bruno MA, Noirhomme Q, Boly M, Laureys $S$, et al. 2012. Granger causality analysis of steady-state electroencephalographic signals during propofol-induced anaesthesia. PLoS One 7:e29072.
Bergmann TO, Molle M, Schmidt MA, Lindner C, Marshall L, Born J, Siebner HR. 2012. EEG-guided transcranial magnetic stimulation reveals rapid shifts in motor cortical excitability during the human sleep slow oscillation. J Neurosci 32:243-253.

Birn RM, Murphy K, Bandettini PA. 2008. The effect of respiration variations on independent component analysis results of resting state functional connectivity. Hum Brain Mapp 29:740-750.

Boly M. 2011. Measuring the fading consciousness in the human brain. Curr Opin Neurol 24:394-400.

Boly M, Coleman MR, Davis MH, Hampshire A, Bor D, Moonen G, et al. 2007. When thoughts become action: an fMRI paradigm to study volitional brain activity in non-communicative brain injured patients. Neuroimage 36:979-992.

Boly M, Faymonville ME, Peigneux P, Lambermont B, Damas P, Del Fiore G, et al. 2004. Auditory processing in severely brain injured patients: differences between the minimally conscious state and the persistent vegetative state. Arch Neurol 61:233-238

Boly M, Faymonville ME, Schnakers C, Peigneux P, Lambermont B, Phillips C, et al. 2008a. Perception of pain in the minimally conscious state with PET activation: an observational study. Lancet Neurol 7:1013-1020.

Boly M, Garrido MI, Gosseries O, Bruno MA, Boveroux P, Schnakers C, et al. 2011. Preserved feedforward but impaired top-down processes in the vegetative state. Science 332:858-862.

Boly M, Massimini M, Tononi G. 2009a. Theoretical approaches to the diagnosis of altered states of consciousness. Prog Brain Res 177:383-398.

Boly M, Phillips C, Balteau E, Schnakers C, Degueldre C, Moonen $\mathrm{G}$, et al. 2008b. Consciousness and cerebral baseline activity fluctuations. Hum Brain Mapp 29:868-874.

Boly M, Seth AK. 2012. Modes and models in disorders of consciousness science. Arch Ital Biol (In press).

Boly M, Tshibanda L, Vanhaudenhuyse A, Noirhomme Q, Schnakers C, Ledoux D, et al. 2009b. Functional connectivity in the default network during resting state is preserved in a vegetative but not in a brain dead patient. Hum Brain Mapp 30:2393-2400.

Boveroux P, Vanhaudenhuyse A, Bruno MA, Noirhomme Q, Lauwick S, Luxen A, et al. 2010. Breakdown of within- and between-network resting state functional magnetic resonance imaging connectivity during propofol-induced loss of consciousness. Anesthesiology 113:1038-1053.

Bressler SL, Seth AK. 2011. Wiener-Granger causality: a well established methodology. Neuroimage 58:323-329.

Bruno MA, Fernandez-Espejo D, Lehembre R, Tshibanda L, Vanhaudenhuyse A, Gosseries O, et al. 2011. Multimodal neuroimaging in patients with disorders of consciousness showing "functional hemispherectomy". Prog Brain Res 193:323-333.

Casali AG, Casarotto S, Rosanova M, Mariotti M, Massimini M. 2010. General indices to characterize the electrical response of the cerebral cortex to TMS. Neuroimage 49:1459-1468.

Chen CC, Henson RN, Stephan KE, Kilner JM, Friston KJ. 2009. Forward and backward connections in the brain: a DCM study of functional asymmetries. Neuroimage 45:453-462.

Coleman MR, Davis MH, Rodd JM, Robson T, Ali A, Owen AM, et al. 2009. Towards the routine use of brain imaging to aid the clinical diagnosis of disorders of consciousness. Brain 132: 2541-2552.

Crick F, Koch C. 1995. Are we aware of neural activity in primary visual cortex? Nature 375:121-123

Cruse D, Chennu S, Chatelle C, Bekinschtein TA, FernandezEspejo D, Pickard JD, et al. 2011. Bedside detection of 
awareness in the vegetative state: a cohort study. Lancet 378:2088-2094.

Davey MP, Victor JD, Schiff ND. 2000. Power spectra and coherence in the EEG of a vegetative patient with severe asymmetric brain damage. Clin Neurophysiol 111:1949-1954.

Dehaene S, Changeux JP. 2005. Ongoing spontaneous activity controls access to consciousness: a neuronal model for inattentional blindness. PLoS Biol 3:e141.

Dehaene S, Changeux JP. 2011. Experimental and theoretical approaches to conscious processing. Neuron 70:200-227.

Dehaene S, Changeux JP, Naccache L, Sackur J, Sergent C. 2006. Conscious, preconscious, and subliminal processing: a testable taxonomy. Trends Cogn Sci 10:204-211.

Del Cul A, Baillet S, Dehaene S. 2007. Brain dynamics underlying the nonlinear threshold for access to consciousness. PLoS Biol 5:e260.

Di HB, Yu SM, Weng XC, Laureys S, Yu D, Li JQ, et al. 2007. Cerebral response to patient's own name in the vegetative and minimally conscious states. Neurology 68:895-899.

Fernandez-Espejo D, Bekinschtein T, Monti MM, Pickard JD, Junque C, Coleman MR, et al. 2011. Diffusion weighted imaging distinguishes the vegetative state from the minimally conscious state. Neuroimage 54:103-112.

Fernandez-Espejo D, Junque C, Cruse D, Bernabeu M, RoigRovira T, Fabregas N, et al. 2010. Combination of diffusion tensor and functional magnetic resonance imaging during recovery from the vegetative state. BMC Neurol 10:77.

Ferrarelli F, Massimini M, Sarasso S, Casali A, Riedner BA, Angelini G, et al. 2010. Breakdown in cortical effective connectivity during midazolam-induced loss of consciousness. Proc Natl Acad Sci U S A 107:2681-2686.

Fitzgerald RD, Lamm C, Oczenski W, Stimpfl T, Vycudilik W, Bauer H. 2001. Direct current auditory evoked potentials during wakefulness, anesthesia, and emergence from anesthesia. Anesth Analg 92:154-160.

Fox MD, Snyder AZ, Vincent JL, Corbetta M, Van Essen DC, Raichle ME. 2005. The human brain is intrinsically organized into dynamic, anticorrelated functional networks. Proc Natl Acad Sci U S A 102:9673-9678.

Friston KJ, Harrison L, Penny W. 2003. Dynamic causal modelling. Neuroimage 19:1273-1302.

Frith C, Perry R, Lumer E. 1999. The neural correlates of conscious experience: an experimental framework. Trends Cogn Sci 3:105-114.

Frith CD. 2011. What brain plasticity reveals about the nature of consciousness: commentary. Front Psychol 2:87.

Garrido MI, Kilner JM, Kiebel SJ, Friston KJ. 2007. Evoked brain responses are generated by feedback loops. Proc Natl Acad Sci U S A 104:20961-20966.

Giacino JT, Ashwal S, Childs N, Cranford R, Jennett B, Katz DI, et al. 2002. The minimally conscious state: definition and diagnostic criteria. Neurology 58:349-353.

Heinke W, Kenntner R, Gunter TC, Sammler D, Olthoff D, Koelsch S. 2004. Sequential effects of increasing propofol sedation on frontal and temporal cortices as indexed by auditory event-related potentials. Anesthesiology 100:617-625.

Honey CJ, Kotter R, Breakspear M, Sporns O. 2007. Network structure of cerebral cortex shapes functional connectivity on multiple time scales. Proc Natl Acad Sci U S A 104:10240-10245.

Horovitz SG, Braun AR, Carr WS, Picchioni D, Balkin TJ, Fukunaga $M$, et al. 2009. Decoupling of the brain's default mode network during deep sleep. Proc Natl Acad Sci U S A 106:11376-11381.
Ilmoniemi RJ, Virtanen J, Ruohonen J, Karhu J, Aronen HJ, Naatanen R, et al. 1997. Neuronal responses to magnetic stimulation reveal cortical reactivity and connectivity. Neuroreport 8:3537-3540.

Jones TB, Bandettini PA, Birn RM. 2008. Integration of motion correction and physiological noise regression in fMRI. Neuroimage 42:582-590.

Kane NM, Moss TH, Curry SH, Butler SR. 1998. Quantitative electroencephalographic evaluation of non-fatal and fatal traumatic coma. Electroencephalogr Clin Neurophysiol 106:244-250.

King JR, Bekinschtein T, Dehaene S. 2011. Comment on "Preserved feedforward but impaired top-down processes in the vegetative state". Science 334:1203; author reply 1203.

Kobylarz EJ, Schiff ND. 2005. Neurophysiological correlates of persistent vegetative and minimally conscious states. Neuropsychol Rehabil 15:323-332.

Koike T, Kan S, Misaki M, Miyauchi S. 2011. Connectivity pattern changes in default-mode network with deep non REM and REM sleep. Neurosci Res 69:322-330.

Kroeger D, Amzica F. 2007. Hypersensitivity of the anesthesia-induced comatose brain. J Neurosci 27:10597-10607.

Ku SW, Lee U, Noh GJ, Jun IG, Mashour GA. 2011. Preferential inhibition of frontal-to-parietal feedback connectivity is a neurophysiologic correlate of general anesthesia in surgical patients. PLoS One 6:e25155.

Lamme VA. 2006. Towards a true neural stance on consciousness. Trends Cogn Sci 10:494-501.

Laureys S. 2005. The neural correlate of (un)awareness: lessons from the vegetative state. Trends Cogn Sci 9:556-559.

Laureys S, Faymonville ME, Degueldre C, Fiore GD, Damas P, Lambermont $B$, et al. 2000. Auditory processing in the vegetative state. Brain 123(Pt 8):1589-1601.

Laureys S, Faymonville ME, Peigneux P, Damas P, Lambermont B, Del Fiore G, et al. 2002. Cortical processing of noxious somatosensory stimuli in the persistent vegetative state. Neuroimage 17:732-741.

Laureys S, Lemaire C, Maquet P, Phillips C, Franck G. 1999. Cerebral metabolism during vegetative state and after recovery to consciousness. J Neurol Neurosurg Psychiatry 67:121-122.

Laureys S, Owen AM, Schiff ND. 2004. Brain function in coma, vegetative state, and related disorders. Lancet Neurol 3:537-546.

Lee U, Kim S, Noh GJ, Choi BM, Hwang E, Mashour GA. 2009. The directionality and functional organization of frontoparietal connectivity during consciousness and anesthesia in humans. Conscious Cogn 18:1069-1078.

Litvak V, Komssi S, Scherg M, Hoechstetter K, Classen J, Zaaroor M, et al. 2007. Artifact correction and source analysis of early electroencephalographic responses evoked by transcranial magnetic stimulation over primary motor cortex. Neuroimage 37:56-70.

Lovstad M, Froslie KF, Giacino JT, Skandsen T, Anke A, Schanke AK. 2010. Reliability and diagnostic characteristics of the JFK coma recovery scale-revised: exploring the influence of rater's level of experience. J Head Trauma Rehabil 25:349-356.

Markowitsch HJ, Kessler J. 2000. Massive impairment in executive functions with partial preservation of other cognitive functions: the case of a young patient with severe degeneration of the prefrontal cortex. Experimental brain research 133:94-102.

Martuzzi R, Ramani R, Qiu M, Rajeevan N, Constable RT. 2010. Functional connectivity and alterations in baseline brain state in humans. Neuroimage 49:823-834.

Massimini M, Boly M, Casali A, Rosanova M, Tononi G. 2009. A perturbational approach for evaluating the brain's capacity for consciousness. Prog Brain Res 177:201-214. 
Massimini M, Ferrarelli F, Esser SK, Riedner BA, Huber R, Murphy M, et al. 2007. Triggering sleep slow waves by transcranial magnetic stimulation. Proc Natl Acad Sci U S A 104:8496-8501.

Massimini M, Ferrarelli F, Huber R, Esser SK, Singh H, Tononi G. 2005. Breakdown of cortical effective connectivity during sleep. Science 309:2228-2232.

Massimini M, Ferrarelli F, Murphy M, Huber R, Riedner B, Casarotto $S$, et al. 2010. Cortical reactivity and effective connectivity during REM sleep in humans. Cogn Neurosci 1:176-183.

Massimini M, Ferrarelli F, Sarasso S, Tononi G. 2012. Cortical mechanisms of loss of consciousness: insight from TMS/ EEG studies. Arch Ital Biol (In press).

Mataro M, Jurado MA, Garcia-Sanchez C, Barraquer L, CostaJussa FR, Junque C. 2001. Long-term effects of bilateral frontal brain lesion: 60 years after injury with an iron bar. Arch Neurol 58:1139-1142.

Monti MM, Vanhaudenhuyse A, Coleman MR, Boly M, Pickard JD, Tshibanda L, et al. 2010. Willful modulation of brain activity in disorders of consciousness. N Engl J Med 362:579-589.

Morishima Y, Akaishi R, Yamada Y, Okuda J, Toma K, Sakai K. 2009. Task-specific signal transmission from prefrontal cortex in visual selective attention. Nat Neurosci 12:85-91.

Murphy M, Bruno MA, Riedner BA, Boveroux P, Noirhomme Q, Landsness EC, et al. 2011. Propofol anesthesia and sleep: a high-density EEG study. Sleep 34:283A-291A.

Murphy M, Riedner BA, Huber R, Massimini M, Ferrarelli F, Tononi G. 2009. Source modeling sleep slow waves. Proc Natl Acad Sci U S A 106:1608-1613.

Nir Y, Fisch L, Mukamel R, Gelbard-Sagiv H, Arieli A, Fried I, et al. 2007. Coupling between neuronal firing rate, gamma LFP, and BOLD fMRI is related to interneuronal correlations. Curr Biol 17:1275-1285.

Nir Y, Mukamel R, Dinstein I, Privman E, Harel M, Fisch L, et al. 2008. Interhemispheric correlations of slow spontaneous neuronal fluctuations revealed in human sensory cortex. Nat Neurosci 11:1100-1108.

Nir Y, Tononi G. 2010. Dreaming and the brain: from phenomenology to neurophysiology. Trends Cogn Sci 14:88-100.

Noirhomme Q, Soddu A, Lehembre R, Vanhaudenhuyse A, Boveroux P, Boly M, et al. 2010. Brain connectivity in pathological and pharmacological coma. Front Syst Neurosci 4:160.

Overgaard M, Overgaard R. 2010. Neural correlates of contents and levels of consciousness. Front Psychol 1:164.

Owen AM, Coleman MR, Boly M, Davis MH, Laureys S, Pickard JD. 2006. Detecting awareness in the vegetative state. Science 313:1402.

Perlbarg V, Bellec P, Anton JL, Pelegrini-Issac M, Doyon J, Benali H. 2007. CORSICA: correction of structured noise in fMRI by automatic identification of ICA components. Magn Reson Imaging 25:35-46.

Phillips CL, Bruno MA, Maquet P, Boly M, Noirhomme Q, Schnakers C, et al. 2011. "Relevance vector machine" consciousness classifier applied to cerebral metabolism of vegetative and locked-in patients. Neuroimage 56:797-808.

Pollonini L, Pophale S, Situ N, Wu MH, Frye RE, Leon-Carrion J, et al. 2010. Information communication networks in severe traumatic brain injury. Brain Topogr 23:221-226.

Rosanova M, Gosseries O, Casarotto S, Boly M, Casali AG, Bruno MA, et al. 2012. Recovery of cortical effective connectivity and recovery of consciousness in vegetative patients. Brain (In press)

Ruby P, Caclin A, Boulet S, Delpuech C, Morlet D. 2008. Odd sound processing in the sleeping brain. J Cogn Neurosci 20:296-311.
Russell IF, Wang M. 1996. Isolated forearm technique. Br J Anaesth 76:884-886.

Sadaghiani S, Hesselmann G, Friston KJ, Kleinschmidt A. 2010. The relation of ongoing brain activity, evoked neural responses, and cognition. Front Syst Neurosci 4:20.

Samann PG, Wehrle R, Hoehn D, Spoormaker VI, Peters H, Tully C, et al. 2011. Development of the brain's default mode network from wakefulness to slow wave sleep. Cereb Cortex 21:2082-2093.

Schiff ND. 2010. Recovery of consciousness after brain injury: a mesocircuit hypothesis. Trends Neurosci 33:1-9.

Schnakers C, Ledoux D, Majerus S, Damas P, Damas F, Lambermont B, et al. 2008. Diagnostic and prognostic use of bispectral index in coma, vegetative state and related disorders. Brain Inj 22:926-931.

Schnakers C, Vanhaudenhuyse A, Giacino J, Ventura M, Boly M, Majerus S, et al. 2009. Diagnostic accuracy of the vegetative and minimally conscious state: clinical consensus versus standardized neurobehavioral assessment. BMC Neurol 9:35.

Schreiber T. 2000. Measuring information transfer. Phys Rev Lett 85:461-464.

Schrouff J, Perlbarg V, Boly M, Marrelec G, Boveroux P, Vanhaudenhuyse A, et al. 2011. Brain functional integration decreases during propofol-induced loss of consciousness. Neuroimage 57:198-205.

Seel RT, Sherer M, Whyte J, Katz DI, Giacino JT, Rosenbaum AM, et al. 2010. Assessment scales for disorders of consciousness: evidence-based recommendations for clinical practice and research. Arch Phys Med Rehabil 91:1795-1813.

Seth AK, Barrett AB, Barnett L. 2011. Causal density and integrated information as measures of conscious level. Philos Transact A Math Phys Eng Sci 369:3748-3767.

Seth AK, Dienes Z, Cleeremans A, Overgaard M, Pessoa L. 2008. Measuring consciousness: relating behavioural and neurophysiological approaches. Trends Cogn Sci 12:314-321.

Soddu A, Boly M, Nir Y, Noirhomme Q, Vanhaudenhuyse A, Demertzi A, et al. 2009. Reaching across the abyss: recent advances in functional magnetic resonance imaging and their potential relevance to disorders of consciousness. Prog Brain Res 177:261-274.

Soddu A, Vanhaudenhuyse A, Bahri MA, Bruno MA, Boly M, Demertzi A, et al. 2012. Identifying the default-mode component in spatial IC analyses of patients with disorders of consciousness. Hum Brain Mapp 33:778-796.

Soddu A, Vanhaudenhuyse A, Demertzi A, Bruno MA, Tshibanda L, Di H, et al. 2011. Resting state activity in patients with disorders of consciousness. Funct Neurol 26:37-43.

Soon CS, Brass M, Heinze HJ, Haynes JD. 2008. Unconscious determinants of free decisions in the human brain. Nat Neurosci 11:543-545.

Stamm M, Aru J, Bachmann T. 2011. Right-frontal slow negative potentials evoked by occipital TMS are reduced in NREM sleep. Neurosci Lett 493:116-121.

Supp GG, Siegel M, Hoipp JF, Engel AK. 2011. Cortical hypersynchrony predicts breakdown of sensory processing during loss of consciousness. Curr Biol 21:1988-1993.

Tononi G. 2004. An information integration theory of consciousness. BMC Neurosci 5:42.

Tononi G. 2008. Consciousness as integrated information: a provisional manifesto. Biol Bull 215:216-242.

Tononi G, Koch C. 2008. The neural correlates of consciousness: an update. Ann N Y Acad Sci 1124:239-261.

Vanhaudenhuyse A, Demertzi A, Schabus M, Noirhomme Q, Bredart S, Boly M, Phillips C, Soddu A, Luxen A, Moonen G, Laureys 
S. 2011. Two distinct neuronal networks mediate the awareness of environment and of self. J Cogn Neurosci 23:570-578.

Vanhaudenhuyse A, Noirhomme Q, Tshibanda LJ, Bruno MA, Boveroux P, Schnakers C, et al. 2010. Default network connectivity reflects the level of consciousness in non-communicative brain-damaged patients. Brain 133:161-171.

Wagner T, Valero-Cabre A, Pascual-Leone A. 2007. Noninvasive human brain stimulation. Annual review of biomedical engineering 9:527-565.

Zeman A. 2001. Consciousness. Brain 124:1263-1289.
Address correspondence to: Mélanie Boly Coma Science Group Cyclotron Research Centre and Neurology Department University of Liège and CHU Sart Tilman Hospital Allée du 6 aout, B30

Liège 4000 Belgium

E-mail: mboly@ulg.ac.be 